\title{
Detection of auditory signals presented at random times: III*
}

\author{
DAVID M. GREEN, University of California at San Diego, La Jolla, California 92037 \\ and \\ R. DUNCAN LUCE, Institute for Advanced Study, Princeton, New Jersey 08540
}

Reaction times to a pure tone in noise were measured. Throughout, the time from the warning signal to the reaction signal was exponentially distributed, and the signal was response terminated. Response criterion, signal intensity, and mean foreperiod wait were varied. A model that assumes a Poisson sensory transduction, a pulse-activated decision process, and an additive bounded residual process was tested. It was concluded that the assumed decision process was in error. Among the empirical results, the dependence of mean reaction time on signal waits was shown to depend largely on the average wait, not the actual one, and that this relationship between mean reaction time and average stimulus wait increased for strong signals and decreased for weak ones.

This paper, the third in a series, continues our attempt to develop a rigorous analysis of behavior in certain auditory detection experiments which mimic, to a degree, the temporal uncertainty of most actual detection situations. In the two earlier papers we reported data from free-response situations which, by definition, lack both warning lights and lights to mark intervals during which a signal might occur. We analyzed these data in terms of several models, all of which postulated a Poisson sensory process but which differed in their assumptions concerning the decision process, in particular in the memory assumed in the decision process. Although some data, especially the tails of various latency distributions, provided strong support for the Poisson assumption, a marked discrepancy between theory and data in another regard suggested that when two or more Poisson events occur at nearly the same time they are stored in a more complex way than was assumed in any of our models. As mathematical difficulties seemed to block the rapid investigation of better memory assumptions, we elected temporarily to bypass that difficulty by modifying our experimental procedure in to what is, essentially, a simple reaction-tine design.

The major differences between our procedures and those employed in usual reaction-time experiments are: (1) the signal may be difficult to detect; (2) once the signal is on, it remains on until the $S$

* This research was supported by Grant GB14049 from the National Science Foundation. The experimentation was carried out on computer facilitics supported by Grant NB07454 from the National Institutes of Health. We are deeply indebted to Miss Virginia L. Maier who prepared the programs needed to run the experiments and for the programs used to analyze the data responds (this has the advantage that the model is much simpler to analyze because there is but one discontinuity in the Poisson parameter rather than two occurring at the onset and offset of the fixed duration signal); and (3) the foreperiod, i.e., the time between the warning signal and the onset of the reaction signal, is a random variable with an exponential distribution. In our second paper, we reported an analysis of such an experiment in terms of a simple Poisson model. Although the model appeared useful in several respects, the data suggested a difficulty in our representation of the S's response criterion. Here, we report new data on this point which clearly reject our previous assumption and, equally clearly, suggest a new one, just as simple.

Our general approach is to treat the observed reaction as a result of two independent stages or processes, and thus the reaction-time distribution becomes a convolution of the times required by each of these two processes. In the middle section of the paper we use numerical Fourier analysis to achieve an analytic separation of these two processes. The results are mixed but it seems clear that, although certain assumptions of the model are surely in error, the model has considerable merit. Finally, the concluding section of the paper concerns signal levels so high that detection is hardly a problem, and so these experiments closely resemble conventional reaction-time experiments. Several characteristics of the resulting reaction-time distributions are studied as a function of two variables: the intensity of the signal and the mean (exponential) foreperiod. The same Poisson model continues to be useful, although, once again, a clear inadequacy is apparent, which suggests that we must modify our assumption about how responses are iniggered.

\section{EXPERIMENTAL PROCEDURE}

Each trial was initiated by a sequence of five "count-down" lights which were lit successively for $200 \mathrm{msec}$ each. (We would have preferred a continuous clock display, but technical problems of synchronization with the computer forced us to a discrete approximation.) Following the offset of the last light, which we refer to as the warning signal, a random wait, $t$, with density, $\lambda e^{-\lambda t}$, preceded the onset of the signal. The $S$ responded by pressing a button which protruded slightly above the armrest. The response terminated the signal, and, after certain data were recorded, a new trial began. If the response preceded the onset of the signal, the signal was blocked.

The exponential delay was programmed as follows. A table of 256 delays was constructed by dividing the exponential density into 256 equiprobable areas and finding the mean of each interval. On each trial, a value was selected at random from this table. Thus, our delays were a discrete approximation to an exponential in which the maximum delay was 6.54 times the mean delay.

The observable times were three. If the response preceded the signal, the only observable time was from the warning signal to the response, the false-alarm time, which we assumed to have a density, denoted $f_{R}(t)$. If the response occurred after signal onset, we recorded both the time from the warning signal to the signal onset, called the signal wait, and the time from the signal onset to the response, called the reaction time. The densities of these times are denoted $f_{S}(t)$ and $f_{R-S}(t)$, respectively. Note that $f_{S}(t) \neq \lambda e^{-\lambda t}$ because $f_{S}$, the signal-wait density, is conditional on the response following the signal and so it favors short waits. On the assumption that observed times in different trials are independent, which probably is not strictly true, we estimated these three densities in the obvious way. For theoretical reasons, given below, we are especially interested in their tails which, for these experiments, we have defined to be all times longer than $1 / 2 \mathrm{sec}$, this choice will receive strong empirical justification.

Each response was followed by feedback as to whether it preceded the signal or, if not, into which of five time categories the reaction time fell. The bounds of these categories were varied from one experiment to another. We tried to locate them so that approximately $20 \%$ of the responses fell in each category. Throughout these experiments, Ss were encouraged to respond as quickly as possible.

A low-level background noise, 40-dB spectrum level, was continuously present in the TDH-39 earphones, which were 


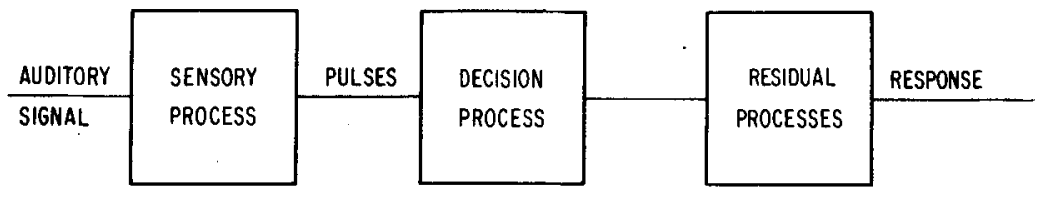

Fig. 1. Functional parts of the general theory.

mounted in sound-absorbing circumaural cushions. The $1,000-\mathrm{Hz}$ sinusoid signal was gated on at a zero crossing. Because signals were response terminated, we can only specify their power, $\mathrm{P}$ (= signal energy per second). The background noise is characterized by its power density, $N_{0}$ (= noise power per cycle per second). To provide some intuitive feel for the quantity reported, we note that a signal-to-noise level of $10 \log \mathrm{P} / \mathrm{N}_{0}=20 \mathrm{~dB}$ corresponds to a level of $10 \log \mathrm{E} / \mathrm{N}_{0}=10 \mathrm{~dB}$ for a signal of $1 / 10-\mathrm{sec}$ duration, and that produces about $75 \%$ detections in a conventional two-alternative forced-choice experiment.

The experiment was controlled by a PDP-9 computer.

The Ss, three undergraduates, were run concurrently in separate sound-treated rooms. They were paid for their services at a rate of $\$ 1.87$ per hour.

\section{REVIEW OF THE MODEL}

The model has been presented in detail in Green and Luce (1967) and Luce and Green (1970), so we only summarize the concepts here. The signal enters into a "sensory process" transducer (see Fig. 1), which converts it into a sequence of discrete "internal events." One may think of these events as neural pulses, although that interpretation is not essential to the model. For a signal, $s$, of constant power, as assume that these events form a Poisson process with a mean rate of $1 / \mu(\mathrm{s})$. An obvious assumption, supported by the data, is that $\mu(\mathrm{s})$ is a strictly increasing function of signal-to-noise ratio. Since we have a constant background and only two signal levels," no signal and signal, in our experiment, we denote these two values of $\mu(\mathrm{s})$ by $v$ and $\mu$, respectively, where $v \leqslant \mu$. We assume that the parameter of the Poisson process changes instantaneously from $v$ to $\mu$ with the onset of the signal. Undoubtedly, this model of the sensory transducer is overly simple. Some transient effects probably occur at the onset and offset of the signal, and when an internal event occurs, some refractoriness may develop, in which case the process cannot be strictly Poisson. In addition, we may (and do, below) find that factors other than the signal-to-noise ratio and the $S$ influence $\mu(\mathrm{s})$; e.g., it may vary with other properties of the experimental situation, such as the density of signal presentations, payoffs, etc. In that case, however, we are likely to conclude, not that the sensory transducer is affected by such variables, but that we have failed to provide an appropriate decomposition of the sensory and decision-making processes.

Next, the events enter into a "decision process" (see Fig. 1) which processes them in some fashion to arrive at a decision about the presence or absence of a signal. Our primary problem is to understand the nature of this process. Since the events arrive sequentially, decisions must of necessity take time, and it is this time, as reflected in the $S$ 's reaction time, that should permit us to get some insight into the nature of the decisions. In our earlier papers, we assumed an exceedingly simple process, which we shall show in this paper cannot be correct. It was assumed that with some probability, $b$, an event is taken seriously, and the first one to be taken seriously initiates a response. The point of this assumption was to model, via changes in the value of the parameter, the effects on response behavior of payoffs, instructions, etc. Given this assumption, the output of the decision process is Poisson, with parameter $b \mu(\mathrm{s})$.

Our final box we label "residual processes" and this box represents all other delays not treated as part of the sensory-decision process. These various delays, though represented by a box at the end of the process, can, of course, occur at any point in the chain. The residual processes might include neural transit times, times to activate muscles, and fixed delays between the sensory and decision process. We lump all of these delays into a single "residual latency" and we assume that it can be described by a bounded random variable with density $r{ }^{1}$ By bounded, we mean that there is some $\tau>0$, such that $r(t)=0$ if either $t \leqslant 0$ or $t \geqslant \tau$. We assume that this process is statistically independent of the two preceding ones.

In essence, then, the overall time is the convolution of the unknown but bounded residual latency with either one or two exponentially distributed latencies-one if the cvent occurs before the signal onset and two if not. Because of the boundedness assumption, it can be shown that all three observable densities have exponential tails; i.e., for $t \geqslant \tau$, the tails are proportional to $\mathrm{e}^{-\alpha \mathrm{t}}$, where $\alpha$ is $\mathrm{b} v+\lambda$ for both $f_{R}$ and $f_{S}$ and is $b \mu$ for $f_{R}-S$. Thus, if the observed tails are exponential, they can be used to estimate $b v$ and $b \mu$. And so, were the theory correct, we should find the ratio $b \mu / b v$ to be independent of $b$ and so of anything that varies the false-alarm rate. The ratio was estimated in the previous study and it appeared to decrease somewhat, about $20 \%$ for one $\mathrm{S}$ and $30 \%$ for the other, with increases in the false-alarm rate, but, statistically, those data were inconclusive. Our new data allow no doubt; happily, they also suggest how to modify the model. We turn to these data.

VARIABLE FALSE-ALARM RATE

In this experiment we simply asked the Ss to vary their "criterion" for reporting the presence of a signal. All other features of the experiment were held constant, in particular $10 \log \mathrm{P} / \mathrm{N}_{0}=10 \mathrm{~dB}$ and $\lambda=0.25 \mathrm{sec}^{-1}$. We generated a more extreme range of false-alarm proportions than we had before, namely, $0.02,0.33$, $0.50,0.60$, and 0.75 . Our training technique was to state a desired proportion and after each 10-min run to report to the $S$ the actual fraction. After about $2 \mathrm{~h}$ of practice they were able to stabilize on a preassigned proportion within binomial variability. There was an initial tendency, especially apparent at the higher false-alarm rates, for the $S$ to achieve the requested proportion by responding to the warning signal as often as needed. These quick false alarms were easily noted and were discouraged; we emphasized that the rate should be varied by "changing the criterion for saying that a signal is present." Stable and acceptable data were ultimately obtained at all five levels.

Estimating the parameters from the tails of the observed densities, we find that the $\mathrm{b} \mu / \mathrm{b} v$ ratio varies from between 10 and 20 at the lowest false-alarm rate to about 1 at the highest. Clearly, the model is incorrect.

Implicitly, we have just assumed that the tails of the distributions are exponential; otherwise, our estimated parameters make little sense. In the previous paper, we showed this to be approximately correct for moderate false-alarm rates, but it continues to be suspect for the high rates we have obtained here. Even if the sensory process is Poisson, if the high false-alarm rates are created by a self-generated process, it would not be particularly surprising for the latter process to deviate significantly from the Poisson.

To test whether or not the tails were exponential, we used the estimated exponential parameter to divide the tail of the false-alarm distribution into 20 


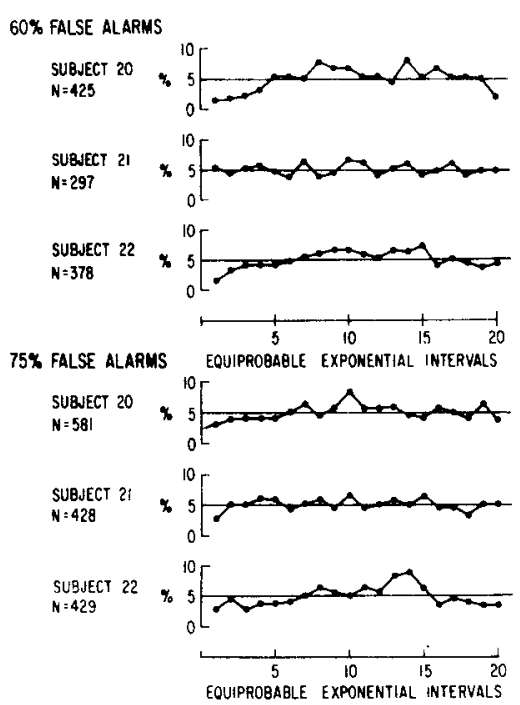

Fig. 2. Observed distribution of responses in the tail of the false-alarm distribution ( $t>1 / 2 \mathrm{sec}$ ) has been fit by an exponential parameter it is divided into 20 equiprobable intervals. Thus we would expect $5 \%$ of the response in each interval. The actual percentage of responses ( $N=$ total number of responses) is shown for three Ss at the two higher false-alarm rates.

equiprobable exponential intervals. Figure 2 presents the actual percentages of times found in these intervals at the two highest false-alarm rates. The data for all three Ss suggest no systematic departure from a horizontal $5 \%$ line, indicating that the Poisson assumption is a good first approximation. The more sensitive $\chi^{2}$ test should have an expected value of about 19 and any value beyond 36 is significant at the 0.01 level. The observed values are shown in Table 1. At least two of the six conditions for $60 \%$ and $75 \%$ rates are too variable. For the two lower rates there are less data and plots, such as Fig. 2, which show considerable variability. The $x^{2}$ values for the two lower false-alarm rates conditions are tou variable.

\section{Modification of the Model}

In the theory stated above (with $b=1$ ), the probability of a false alarm is distribution. Once the tail of the are also given in Table 1; four of the six

$$
\mathrm{P}(\mathrm{S}>\mathrm{R})=\frac{v}{v+\lambda} \mathrm{R}_{-\lambda},
$$

where

$$
\mathrm{R}_{-\lambda}=\int_{0}^{\infty} \mathrm{e}^{-\lambda \mathrm{t}} \mathrm{r}(\mathrm{t}) \mathrm{dt}
$$

Since $\mathrm{R}, \lambda \leqslant 1$, the maximum possible probability is $v /(v+\lambda)$. If false sensory detections occur at a mean rate of at least one per $10 \mathrm{sec}$, i.e., $v \leqslant 0.1 \mathrm{sec}^{-1}$, then for $\lambda=0.25 \mathrm{sec}^{-1}$ the largest possible false-alarm proportion is about 0.3 . An $S$ faced with a demand for a higher proportion can, of course, simply press the response button, independent of any detection process. The easiest way to do this, as all Ss did initially, is to respond inmediately to the warning light on the appropriate proporion of trials. Such behavior is analogous to what Ollman (1966) and Yellot (1967) have called "fast guesses" in their studies of choice reaction time.

We conjecture that, when discouraged from making fast guesses, an $S$ may somehow generate an internal random process, independent of the sensory process, and that he simply "adds" the two together to create a single Poisson process which, when an event occurs, triggers the response process. We think of the background sensery process as having the parameter $v_{0}$ in the case of noise alone. and as having the parameter $v_{0}+\rho$ when the signal is added to the noise. Assume the self-generated process is Poisson and has paramter $\sigma$, then the estimated parameters are $v=v_{0}+\sigma$ for noise alone, and $\mu=v_{0}+\rho+\sigma=v+\rho$ for signal plus noise. The parameters $v_{0}$ and $\rho$ should depend on the stimulus conditions, for example the noise background, $N_{0}$, and the signal level, $P$, and he independent of instructions. The parameter o should vary with instructions but be independent of the stimulus conditions. If lliese assumptions are true, then both $v$ and $\mu$ will vary with instructions but $\mu-v=\rho$ will not. The mathematical forms of the distribution remain unchanged; we simply set $\mathrm{b}=1$ and assume that $v$ and $\mu$ will vary with changes in the false-alarm rate. The estimates of $\mu-v$, however, should be independent of changes in the false-alarm rate.

Table 1

$\chi^{2}$ and Sample Sizes for Exponential fit to Tails $(t \geqslant t / 2)$ of the Reaction-Time Distribution

\begin{tabular}{|c|c|c|c|c|c|c|c|c|}
\hline \multirow[b]{3}{*}{ Subject } & \multicolumn{8}{|c|}{ Ialie-Alarm Rate } \\
\hline & \multicolumn{2}{|c|}{$75 \%$} & \multicolumn{2}{|c|}{$60 \%$} & \multicolumn{2}{|c|}{$50 \%$} & \multicolumn{2}{|c|}{$33 \%$} \\
\hline & $\chi^{2}$ & $\mathrm{~N}$ & $x^{2}$ & $N$ & $x^{2}$ & $\mathrm{~N}$ & $x^{2}$ & $N$ \\
\hline 20 & 22.1 & 581 & 57.1 & 405 & 87.3 & 324 & 92.9 & 190 \\
\hline 21 & 15.0 & 428 & 9.5 & 297 & 28.7 & 276 & 40.9 & 209 \\
\hline 22 & 48.1 & 429 & 29.5 & 378 & 25.9 & 281 & 53.9 & 202 \\
\hline
\end{tabular}
for the Four Highest false-Alarm Rates
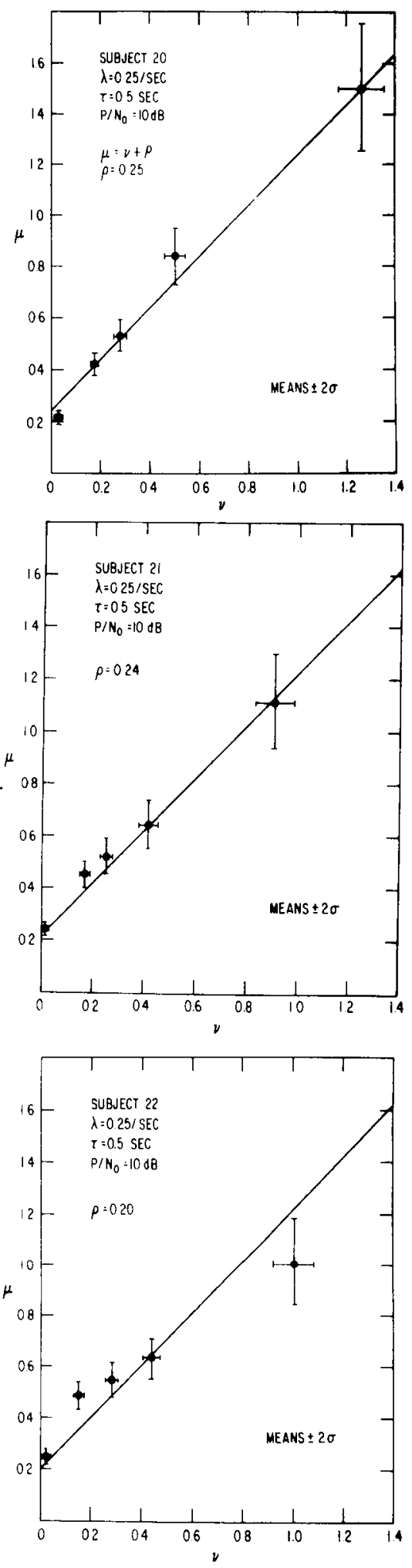

Fig. 3(abc). Estimates of parameters $\mu$ and $v$ for five different false-alarm proportions at a fixed signal-to-noise ratio for three Ss. The parameter $\mu$ is estimated from the tail of the reaction-time distribution. The parameter $v$ is estimated from the tails of the stimulus-wait and false-alarm distributions. 


\section{Results}

Figure 3 presents the plots of $\mu$ vs $v$ for the three Ss. If our new assumption is correct, these should be linear with a slope of one, which is approximately so. The estimated values of $\rho$ are $0.25,0.24$, and 0.20 .

We cannot be sure how generally true our new hypothesis is. Certainly it is conceivable that were the mean wait for the signal much greater than the $4 \mathrm{sec}$ of this experiment, an $S$ might find it impossible to generate an appropriate Poisson process. We shall not worry about it, however, since later results suggest that we have not yet achieved an appropriate decomposition into sensory and decision processes, in which case the relation $\mu=v+\rho$ is but an approximation to something else.

\section{DISTRIBUTION OF THE RESPONSE PROCESS}

\section{General Approach}

As we pointed out earlier (see Fig. 1), our model postulates that the overall observable reaction time is the sum of two independent unobservable latencies-one is the time it takes the decision process to act on the Poisson input and the other is all other delays in the process, their sum being called the "residual latency." Let $f$ denote the observable reaction-time density. Since $f$ is, by assumption, the sum of two independent latencies, call them $s$ for the sensory-decision process and $\mathrm{r}$ for the residual process, we may write $f$ as a convolution of $s$ and $r$.

$$
f(t)=\int_{0}^{t} s(x) r(t-x) d x
$$

The Fourier transform of $f$ is defined by

$$
F(\omega)=\int_{0}^{\infty} e^{-i \omega t} f(t) d t, \quad i=(-1)^{1 / 2}
$$

(we write the lower limit of integration as 0 rather than $-\infty$ because all of our densities are 0 to $t \leqslant 0$ ). If $S$ and $R$ denote, respectively, the Fourier transforms of $s$ and $r$, it is easy to show that

$$
F(\omega)=S(\omega) R(\omega)
$$

(Various other classical transformations, including the LaPlace, have this important property of converting a convolution into a multiplication.)

A particular theory of the sensory-decision process allows one to describe $s$ and, hence, $S$. If the parameters of this description can be estimated in some manner, either from signal-to-noise measurements or from the reaction-time data itself, then, using Eq. 3, one can divide $F$ by $S$ and thus estimate $R$. Inverting $R$ leads to an estimate of $r$.

In practice, we work with conditional densities and the resulting equations are somewhat more complex, but the basic ideas are the same. Because we assume that the latency of the response process is bounded, we can estimate the two sensory parameters, $v$ and $\mu$, from the tails of the observed distributions. The parameter $\lambda$, which is controlled by the $E$, and the quantity $R_{-} \lambda$, which we may estimate from $v, \mu$, and the observed values of the tail probabilities, complete our description of the S process. We then take the Fourier transform of the observed reaction-time distribution and attempt to estimate $R$, and so $r$.

Such an estimate of $r$ permits us to test at least two aspects of the theory. First, we can see if our assumed bound $\tau$ on the response process is confirmed. Second, and much deeper, we can see if the estimate of $r$ is independent of our experimental conditions-for example, is $r$ independent of signal strength, mean wait for a signal, and instructions about false-alarm rates? These tests will be made, but first we must obtain the exact form for Eq. 3 and we must discuss some of the practical realities of calculating Fourier transforms from estimates of probability densities.

\section{Specific Equations}

We denote functions and transforms by

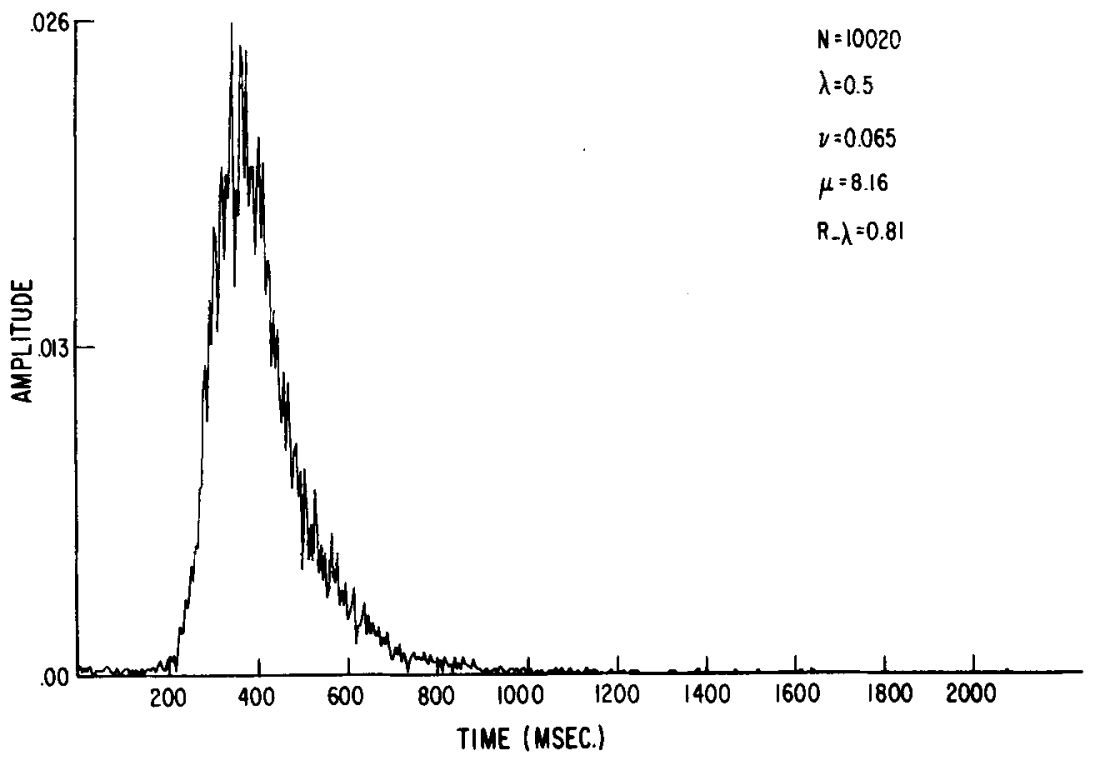

Fig. 4. Observed reaction-time distribution for one $\mathbf{S}$. The signal-to-noise level was fixed $\left(10 \log P / N_{0}=21\right)$ and the average exponential wait was $2 \sec \left(\lambda=0.5 \sec ^{-1}\right)$. The $y$ axis is simply the observed proportion of responses in successive intervals approximately $5 \mathrm{msec}$ in width. The total number of responses is about 10,000 . The various parameters associated with this distribution are discussed in the text. 


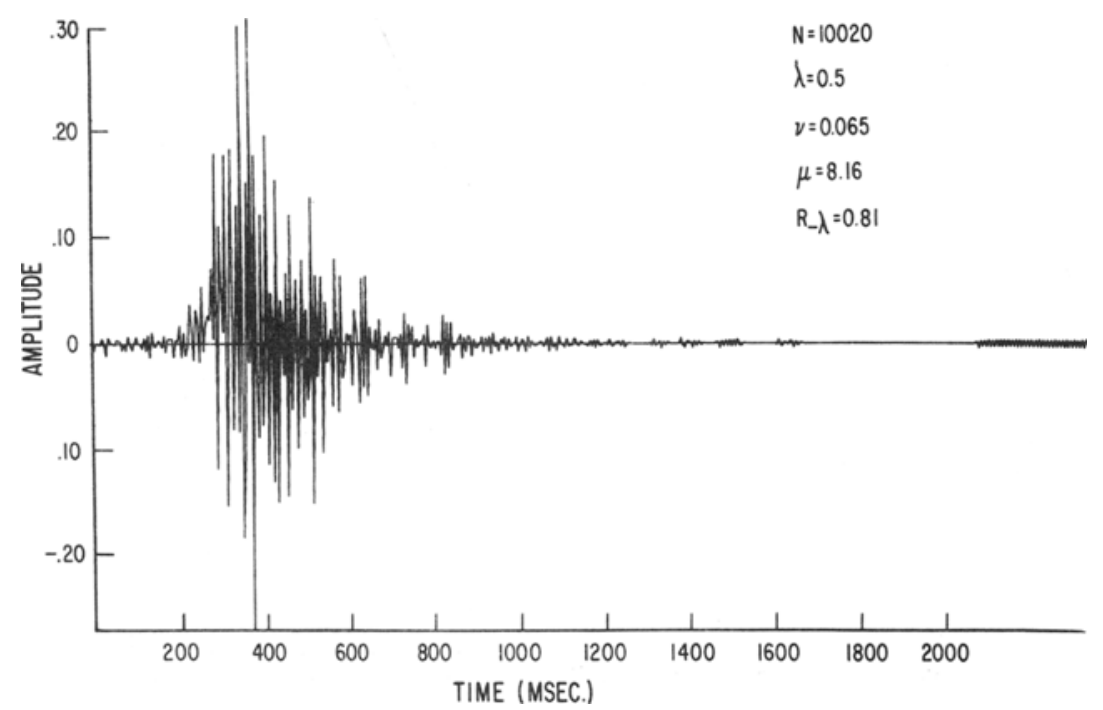

Fig. 5. The estimate of $r(t)$, based on the data given in Fig. 4, and a straightforward application of Fourier transforms, Eq. 5, and inverting $R(\omega)$ to find $r(t)$.

to calculate the transform; however, a certain trick of rewriting can be exploited to reduce the number to the order of $N \log _{10} N$. The savings are enormous. namely, $\mathrm{N} / \log _{10} \mathrm{~N}$, which for $\mathrm{N}=10^{3}$ is a factor of about 300 . This discovery. made a few years ago, has made the numerical calculation of Fourier transforms practical on contemporary computers. The program to do so is called the Fast Fourier Transform, or FFT. All of our calculations use this method.

\section{Chaos in the Calculations}

Were there no problems of convergence in the series used to approximate the integral in Eq. 2, the calculations based on Eqs. 4-6 would be routine. But there are problems, as we now illustrate. The histogram shown in Fig. 4 is based on about 10,000 observations grouped into approximately 5 -msec intervals. Using the tails of this and the corresponding $f_{R}$ and $f_{S}$ frequency functions, we estimate $v=0.065$ and $\mu=8.16$. From the theory, one finds that the areas in the tails of $f_{R}$ and $\mathrm{f}_{\mathrm{S}}$ are functions of $v$ and $\mu$ and the two constants $R_{-} \lambda$ and $R_{v}$. Solving, we estimate $R_{-} \lambda=0.81$. Substituting these estimates in Eq. 5, solving for $R$ and inverting to estimate $r$, yiclds the mess shown in Fig. 5-hardly a good approximation to a density function. Similar computations based on Eqs. 4 and 6 produced even less encouraging results: the chaos in those cases is badly aggravated by the fact that the inverted function las to be multiplied by $e^{\lambda t}$ to get $r$.

T. $0^{*}$ anyone familiar with Fourier transforms, the high-frequency fluctuations in Fig. 5 suggest the prescnce of rapid waveform where the derivative is extremely large. Of course, an empirical histogram is the quintessence of a discontinuous function since each interval of the distribution may produce an abrupt thange in the function. Perhaps, then, we should attempt to smoth our data in some fashion so as to minimize the irrelevant, but noticeable, effects of random discontinuitics. Of course, any smoothing of the data necessarily induces a corresponding blur in our estimate of $r$ because averaging renders indistinct some $\lambda=0.5 \mathrm{sec}^{-1}$. temporal events that formerly were distinct. The question is: Just how high is the price, in terms of loss of resolution, of reducing oscillations in the estimate of $r$ by smoothing the data? To gain some idea, we analyze carefully the simplest temporal event and the simplest of discontinuities, the impulse.

\section{Smoothing an Impulse}

Suppose that all response latencies have the same value $t_{0}$, which is equivalent to saying that all of the density $r$ is concentrated at a single time, namely $t_{0}$. It is easy to see from Eq. 36 in Luce and Green (1970) that

$$
\mathrm{f}_{\mathrm{R}-\mathrm{S}}(\mathrm{t})= \begin{cases}\frac{v}{\theta} \mathrm{e}^{-\lambda\left(\mathrm{t}_{0}-\mathrm{t}\right)}, & 0 \leqslant \mathrm{t} \leqslant \mathrm{t}_{0} \\ \frac{\mu}{\theta} \mathrm{e}^{-\mu\left(\mathrm{t}-\mathrm{t}_{0}\right)}, & \mathrm{t}_{0} \leqslant \mathrm{t}\end{cases}
$$

where, as before,

$$
\theta=\frac{v}{\lambda}\left(1-\mathrm{R}_{-\lambda}\right)+1=\frac{v}{\lambda}\left(1-\mathrm{e}^{-\lambda t_{0}}\right)+1
$$

Figure 6 shows an approximation to this density when $\mathfrak{t}_{0}$ is $240 \mathrm{msec}$ and $v$, $\mu$, and $R_{-} \lambda$ have the values indicated in the figure (these were chosen to be roughly those estimated in the data of the two Ss whose data we will present). As one can see, $f_{R-S}$ has an abrupt jump in the region of $t_{0}$. Taking this as our "data," and using 5-msec intervals for the width of our histogram, we use Eq. 5 to recover our estimate of an impulse. The results are shown in Fig. 7. As

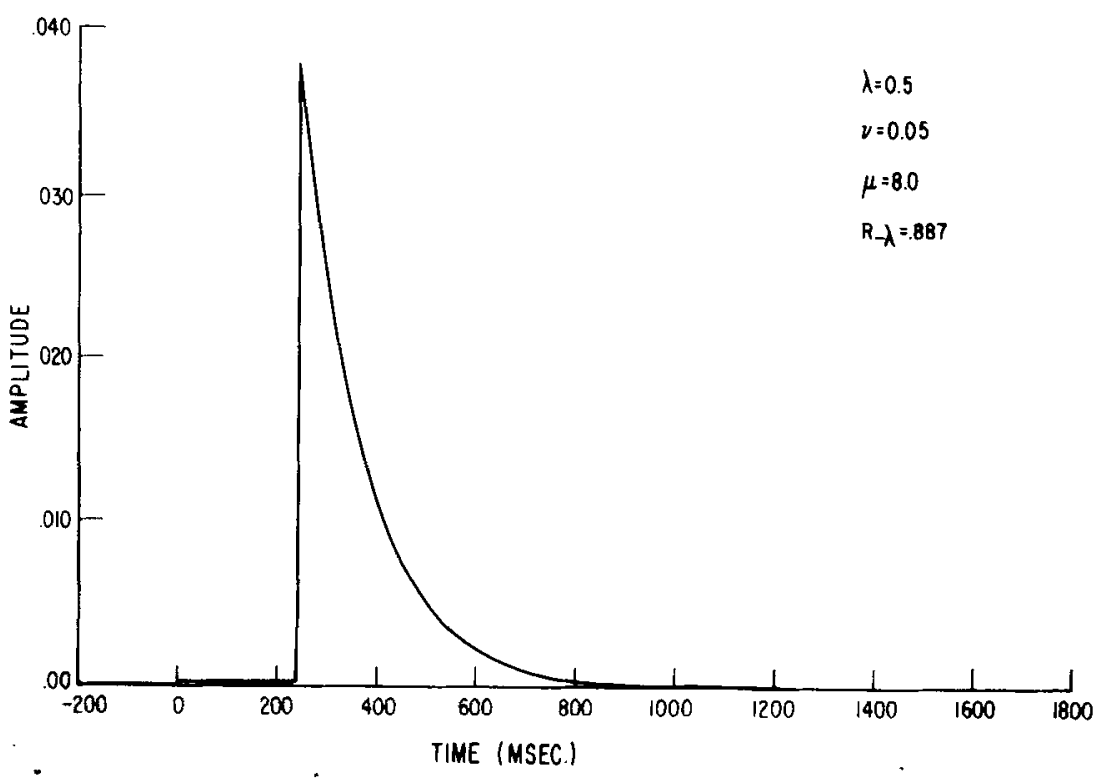

Fig. 6. Expected form for the distribution of reaction times if $r(t)$ were an impulse occurring at $240 \mathrm{msec}$. We assume $\mu=8.0 \mathrm{sec}^{-1}, v=0.05 \mathrm{sec}^{-1}, R_{-} \lambda=0.88$, and 


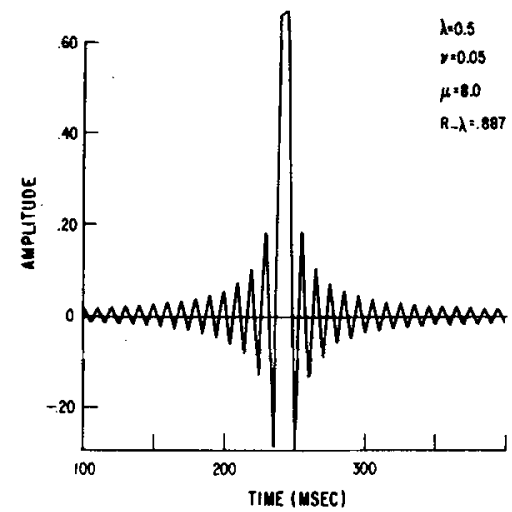

Fig. 7. Estimate of $r(t)$ from the "data" of Fig. 6 using Eq. 5. The parameter values are those given in Fig. 6. Note that we have scaled in order to show that two pulses are recovered in the vicinity of $240 \mathrm{msec}$.

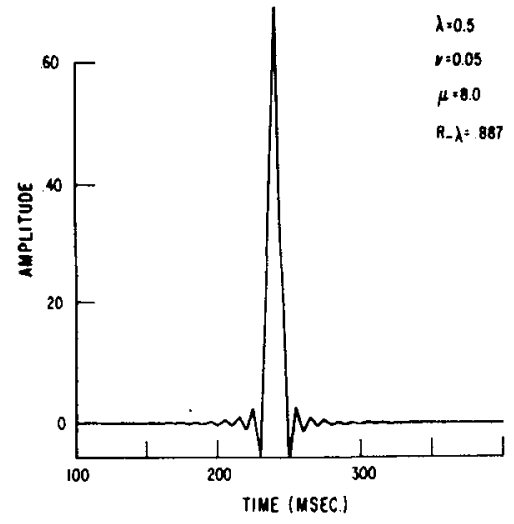

Fig. 8. Same procedure as used in Fig. 7 except that a sliding average is taken of the "data" in Fig. 6 before the transform procedure is applied to the smoothed data. Note the expanded time scale.

expected, we do not recover a single impulse, but a function containing two large positive values in the vicinity of $t_{0}$ and considerable high-amplitude oscillation near that point in time. (Note that the time scale of Figs. 7 and 8 is expanded by a factor of 4 from Fig. 6 in order to show in detail what happens.)

ihis poor estumate is improved dramatically by even the slightest smoothing. For example, suppose we simply average adjacent points in the input histogram. The only visually noticeable change in the histogram is to interpolate a single point midway on the discontinuity; however, the resulting estimate, shown in Fig. 8, is much improved, both in definition and stability.

Such a pronounced effect led us to investigate more extensive running averages. A family of weighting functions, called "Hamming windows" (Kaiser, 1966), have been used successfully by engineers, so we elected to try them. For

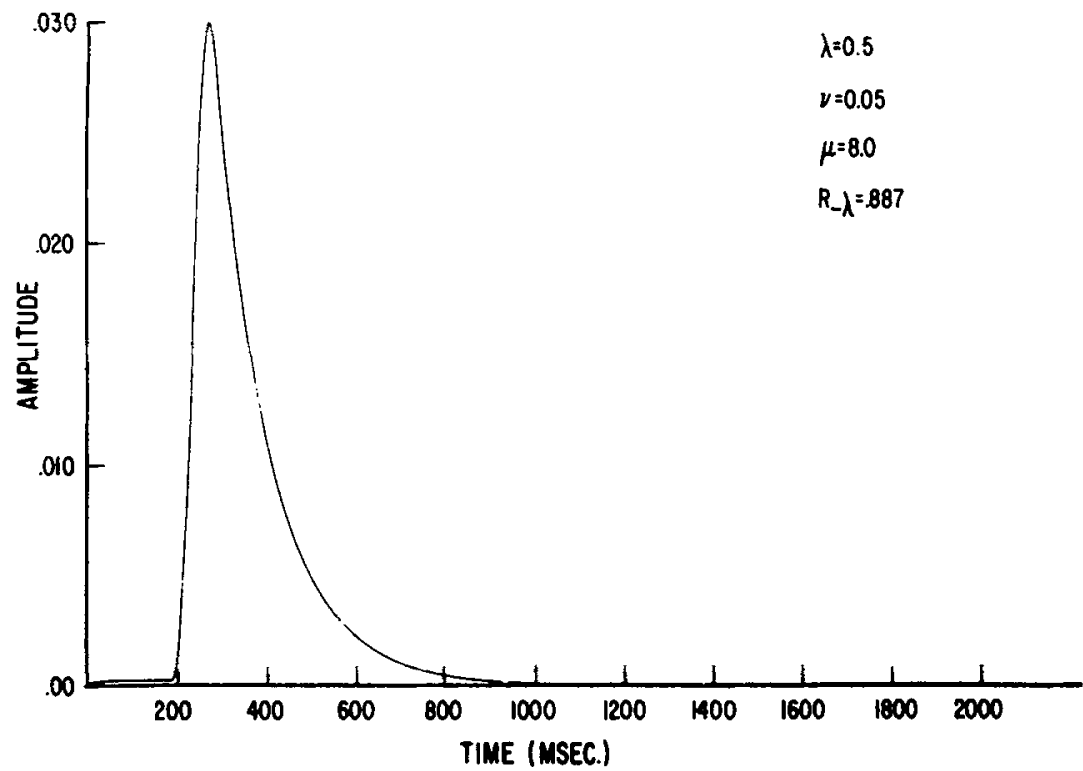

Fig. 9. Hammed version of the impulse response shown in Fig. 6. The smoothing function averages over 21 points and thus extends over a 105 -msec interval.

the data presented in Fig. 4, trial and error suggests that averaging over 21 successive points gives the desired smoothing without sacrificing too much resolution. ${ }^{2}$ For example, "hamming" the impulse in Fig. 6 yields Fig. 9, and that in turn yields the estimate of the impulse (see Fig. 10). Observe that the impulse has been distributed over a considerable band of times, and so when we apply this technique to experimental data we must not forget that only temporal features wider than this "pulse" width will pass our smoothing function.

Obviously, such grossly imperfect temporal resolution imposes serious limits

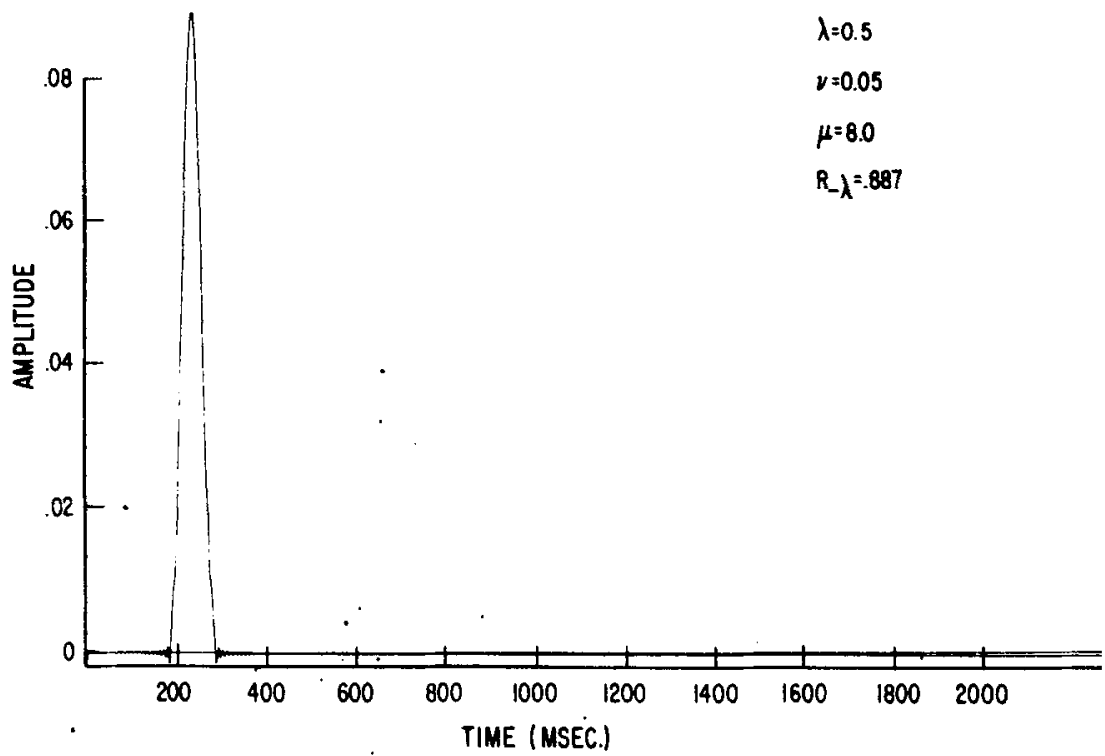

Fig. 10. The impulse recovered from the "data" of Fig. 9. The parameters are the same as given in the legend to Fig. 6 . The peak of the impulse occurs at $240 \mathrm{msec}$, as it should, but is appreciably blurred by the smoothing function. 


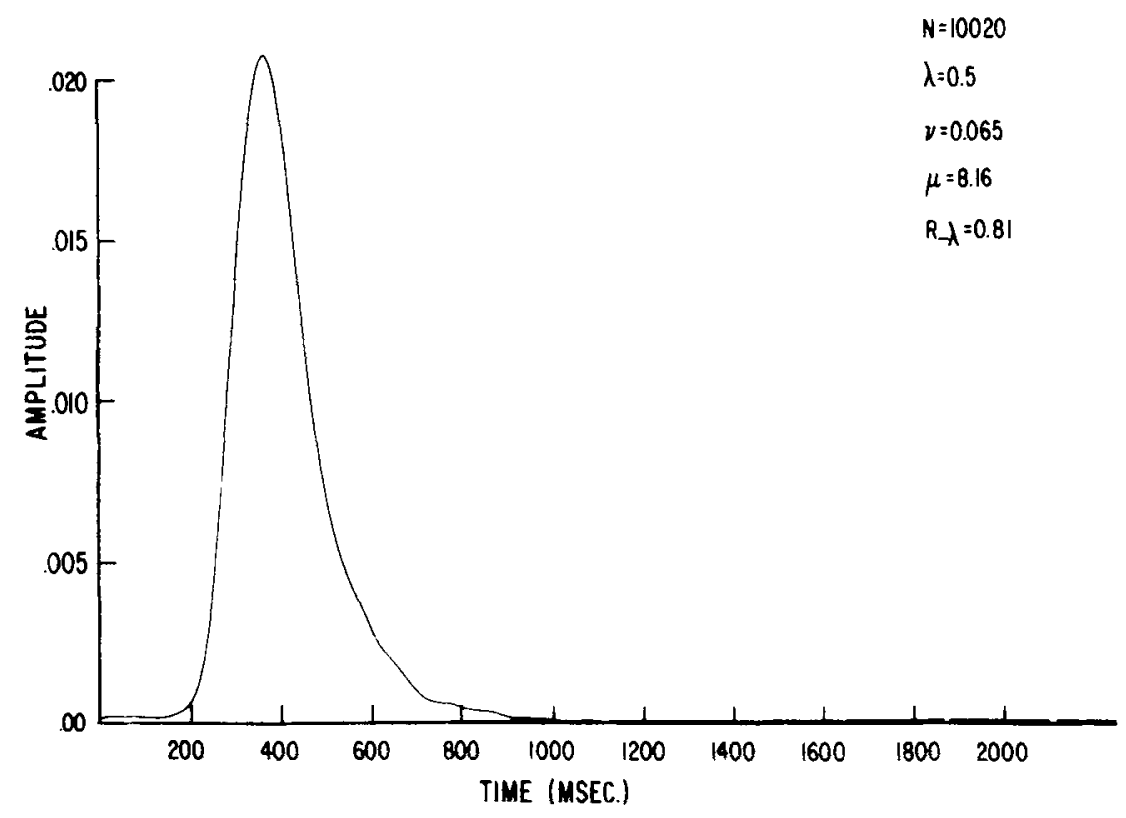

Fig. 11. Hammed version of the data shown in Fig. 4. The smoothing function averages over 21 points and thus produces the blurring of an impulse, as shown in Fig. 10.

$S$ 's reaction times), and we reduced the hamming to 7 points.

\section{Smoothed Data}

Figure 11 is the hammed version of the data shown in Fig. 4, and Fig. 12 shows the resulting estimate of $r$. The only difference between Figs. 5 and 12 is in the smoothing: the equations, parameter values, and the computation of formulas are identical. Figures 13 and 14 show similar data and the corresponding estimate for the second S.

\section{Discussion and Conclusions}

First, the estimate of $r$ is abruptly bounded on the right at slightly more than $400 \mathrm{msec}$ for one $\mathrm{S}$ and $450 \mathrm{msec}$ for the other, which is well inside our guess of $500 \mathrm{msec}$ for $t$. Second, obviously a probability density cannot be negative, yet both Ss exhibit a rather large negative dip following what appears to be the end of the reaction-time distribution. This same general pattern has also been observed in some other data which we are in the process of analyzing. We are fairly certain that it is not an artifact of the computation, but we cannot suggest any simple feature of the theory that is at fault. It probably reflects a rather fundamental deficiency of the theory. Third, ubserve that since $f_{R-S}$ is the convolution of $r$ with something else, $r$ should be more peaked and its mode should be to the left of that of $f_{R-S}$, and this is the case. For the first $S$ (Figs. IJ and 12) it is about $70 \mathrm{msec}(370 \mathrm{msec}$ to $300 \mathrm{msec}$ ). For the second $S$ (Figs. 13 and 14) this strongly rejects our present hypothesis about the sensory-decision process. The argument is straightforward. The distribution of what we call the "residual" process should be independent of signal strength; indeed, this is the main motivation for this analysis. With large signal-to-noise ratios $\left(10 \log \mathrm{P} / \mathrm{N}_{0}\right.$ $=54 \mathrm{~dB}$ ), these same $\mathrm{Ss}$ yield reaction-time distributions with modes of 195 and $240 \mathrm{msec}$, respectively, and very low false-alarm rates. For this signal-to-noise ratio, the value of $\mu$ is so large that, for any reasonable hypothesis about the nature of the decision process, there can be little difference between $r$ and $f_{R-S}$. This forces us to conclude that our estimates of $r$ actually are estimates of $r$ convolved with some aspect of the decision process which takes about $80-100$ msec on the average, the resultant is probably not terribly variable, and is certainly bounded. We are presently exploring another decision model incorporating many of these features.

\section{SIMPLE REACTION TIMES}

In the preceding experiments, the signal was generally difficult to detect and the reaction times were slow, often a second or longer. Increasing the signal intensity converts one of these detection experiments into something more properly called a reaction-time experiment with a random foreperiod. The procedure is, however, still slightly unorthodox because the signal is not of fixed, short duration, but rather is response terminated.

We have yet to devise a satisfactory way to estimate the sensory parameters $\mu$ and $v$ for loud signals. Large $\mu$ s are difficult to $N=10020$

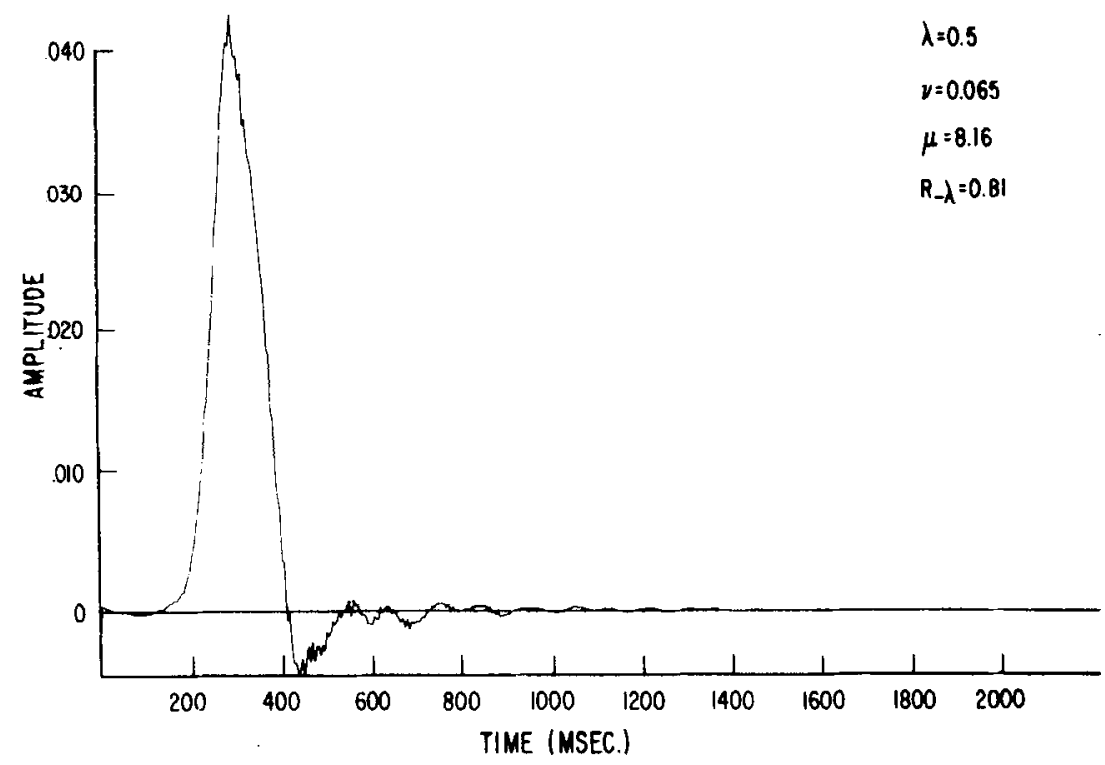

Fig. 12. Application of the Fourier transform techniques to the data of Fig. 11 to estimate $r(t)$. 


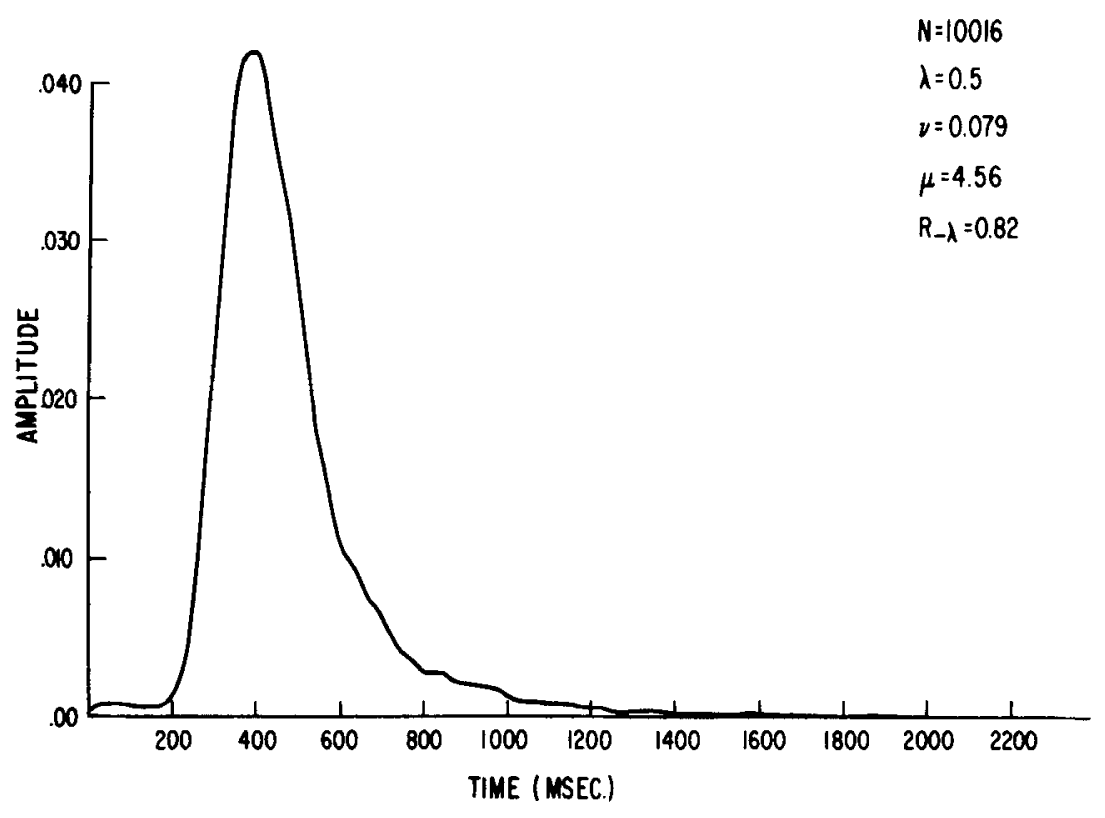

Fig. 13. Hammed data for a second S. Seven points are used in the Hamming window but the original data were based on 12-msec intervals, so the smoothing is roughly comparable to that used for the first $S$.

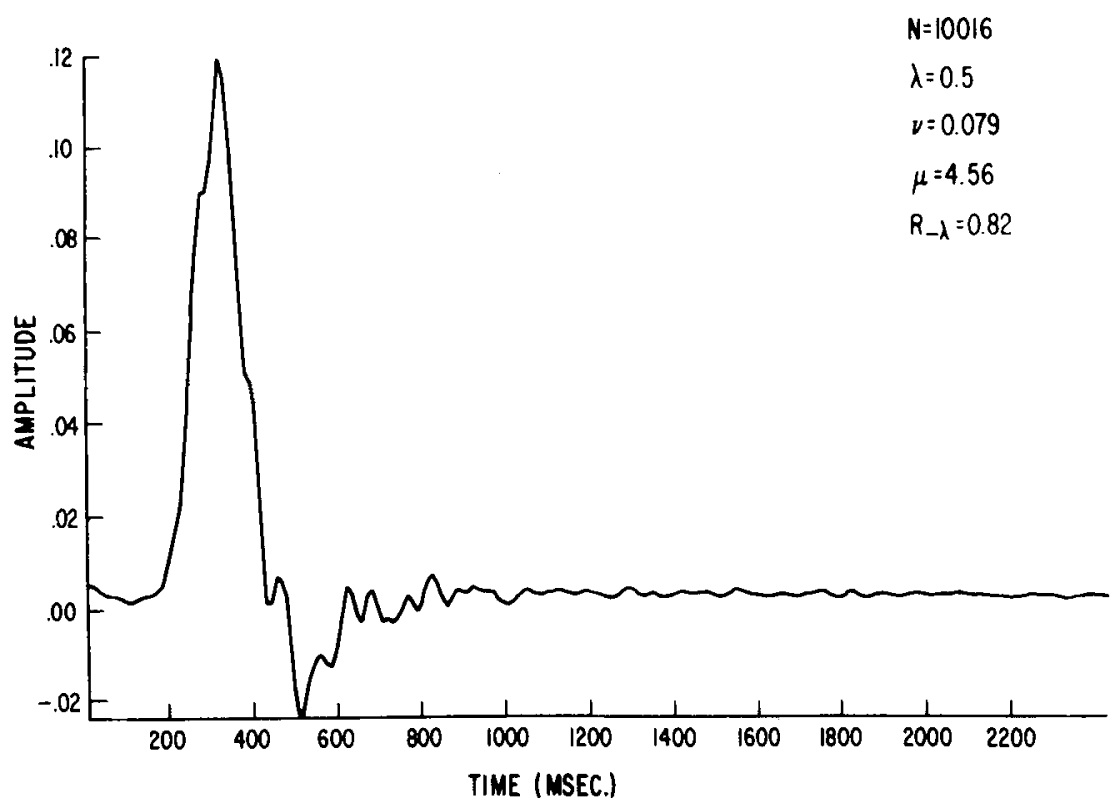

Fig. 14. Application of the Fourier transform techniques to the data of Fig. 13 to estimate $\boldsymbol{r}(\mathbf{t})$.

estimate because long reaction times are infrequent, and thus the tails of the reaction-time distributions are very slight, and, without an extremely precise estimate of $\tau$, which we do not have, there is little hope of estimating $\mu$ uncontaminated by the residual distribution $r$. The problem in estimating $v$ from $\mathrm{f}_{\mathrm{R}}$ is different. We cannot be certain, but we fear (see below), that this distribution is contaminated by a number of anticipatory responses-fast guesses, as they are sometimes called-which are not properly reflected by the Poisson false-alarm source described earlier. Thus, we shall have to be satisfied with examining the data and evaluating qualitative features of the model that do not rest upon knowing $\mu$ and $v$ exactly.

In the following studies, we manipulated three variables: the signal intensity, the mean wait for the signal to occur, $1 / \lambda$, and the false-alarm rate. The interaction of the false-alarm rate, which we manipulated by instructions, with each of the other two is explored. We find that both the signal intensity and the mean wait for the signal produce systematic changes in the mean reaction time. In both experiments, we determined the mean reaction time conditional on the actual wait (rather than on the mean wait) for the signal. It is clear that this relation depends on the intensity of the signal, the mean reaction time increasing with longer waits for high signal levels and decreasing for weak signal levels The data suggest that, in spite of the complexity, the process can be treated in first approximation as stationary in time which is what is predicted by the model.

Signal Intensity and Mean Reaction Time

The five signal intensities ranged from $10 \log \mathrm{P} / \mathrm{N}_{0}=14 \mathrm{~dB}$, which is quite difficult to detect, to $54 \mathrm{~dB}$, which is extremely loud but produces neither startle nor discomfort. We established three false-alarm rates-low, 30\%, and 50\% (see above for the method). In practice, "low" meant about $1 \%$ for all but the lowest signal level, where it was about $5 \%$; for the $30 \%$ condition the range was from $25 \%$ from $33 \%$ over Ss and signal levels; and for the $50 \%$ condition the range was from $45 \%$ to $55 \%$. At all rates we emphasized speed, and in the early practice trials we reported their mean reaction times to the Ss. For all conditions, the mean of the exponential signal wait was $4 \mathrm{sec}$.

The mean reaction times and the numbers of observations on which they are based are shown in Table 2. We note several features of the data. First, given any false-alarm rate, and any $S$, mean reaction time decreases with increasing signal intensity. This trend is consistent with several previous studies (McGill, 1961; McGill \& Gibbon, 1965; Kohfeld, 1968). However, the pattern as a function of false-alarm rate is not simple. In particular, looking only at the $30 \%$ and $50 \%$ rates, the mean reaction time is less for the higher rate, but the magnitude of the decrease depends both on the signal intensity and on the S. For example, at $14 \mathrm{~dB}, \mathrm{~S} 22$ shows a decrease of about $200 \mathrm{msec}(1.227$ to 1.013$)$, whereas, at $54 \mathrm{~dB}$, his decrease is only $27 \mathrm{msec}(221$ to 194). But comparing the data at the low false-alarm rate to the $30 \%$ condition, Ss 20 and 21 exhibit an increase in mean reaction time for all signal intensities, save the lowest. The results for $\mathrm{S} 22$ are very nearly independent of the false-alarm rate except for the lowest signal intensity, where the mean reaction time decreases as false-alarm rate increases.

To evaluate the significance of these differences, one really must know something about the variability of these data. In Fig. 15, we have plotted the standard deviation vs the mean reaction time for all the data reported in this paper. 
Table 2

Mean Reaction Time as a Function of Signal-to-Noise Level and False-Alarm Rate

\begin{tabular}{|c|c|c|c|c|c|c|c|}
\hline & & & & & & & \\
\hline & $0 \log P / N_{0}$ & MRT & $\mathrm{N}$ & MRT & $\mathrm{N}$ & MRT & $\mathrm{N}$ \\
\hline $\begin{array}{c}\text { Low } \\
\text { False-Alarm } \\
\text { Rate }\end{array}$ & $\begin{array}{l}14 \\
24 \\
34 \\
44 \\
54\end{array}$ & $\begin{array}{r}2.899 \\
.362 \\
.296 \\
.264 \\
.258\end{array}$ & $\begin{array}{l}409 \\
556 \\
576 \\
445 \\
453\end{array}$ & $\begin{array}{r}1.884 \\
.318 \\
.258 \\
.237 \\
.223\end{array}$ & $\begin{array}{l}271 \\
549 \\
451 \\
446 \\
344\end{array}$ & $\begin{array}{r}1.936 \\
.319 \\
.253 \\
.244 \\
.220\end{array}$ & $\begin{array}{l}410 \\
552 \\
576 \\
448 \\
455\end{array}$ \\
\hline $\begin{array}{l}\text { Probability of } \\
\text { False Alarm } \\
\text { About } 33 \%\end{array}$ & $\begin{array}{l}14 \\
24 \\
34 \\
44 \\
54\end{array}$ & $\begin{array}{r}1.227 \\
.371 \\
.298 \\
.291 \\
.290\end{array}$ & $\begin{array}{l}302 \\
317 \\
314 \\
313 \\
312\end{array}$ & $\begin{array}{r}1.164 \\
.374 \\
.324 \\
.257 \\
.279\end{array}$ & $\begin{array}{l}288 \\
344 \\
350 \\
359 \\
359\end{array}$ & $\begin{array}{r}1.227 \\
.329 \\
.262 \\
.244 \\
.221\end{array}$ & $\begin{array}{l}371 \\
400 \\
386 \\
327 \\
398\end{array}$ \\
\hline $\begin{array}{l}\text { Probability of } \\
\text { False A larm } \\
\text { About } 50 \%\end{array}$ & $\begin{array}{l}14 \\
24 \\
34 \\
44 \\
54 \\
\end{array}$ & $\begin{array}{r}1.188 \\
.318 \\
.273 \\
.268 \\
.243\end{array}$ & $\begin{array}{l}307 \\
259 \\
246 \\
257 \\
233\end{array}$ & $\begin{array}{l}.915 \\
.343 \\
.236 \\
.248 \\
.236\end{array}$ & $\begin{array}{l}232 \\
230 \\
295 \\
227 \\
206\end{array}$ & $\begin{array}{r}1.013 \\
.294 \\
.251 \\
.227 \\
.194\end{array}$ & $\begin{array}{l}291 \\
286 \\
301 \\
280 \\
258\end{array}$ \\
\hline
\end{tabular}

Note $-\lambda=0.25 \mathrm{sec}^{-1}$; the mean wait for the signal was $4 \mathrm{sec}$.

The rule-of-thumb is that at low signal intensities the ratio of the mean to the standard deviation is about 1 , whereas at high intensities it increases to about 5 . Only the data taken at the lowest signal-to-noise ratio seems to support the decrease in mean reaction time with increase in false-alarm rate. For the higher signal-to-noise ratios the mean reaction is either independent of false-alarm rate or nonmonotonically related. A variety of sequential models, in which the $S$ is postulated to accumulate sensory information until some criterion is reached, would predict that the mean reaction time should decrease as the false-alarm rate increases (Stone, 1960; Grice, 1968; Lamming, 1968). The cxact relation between false-alarm rate and mean reaction time is somewhat difficult to assess for the Poisson model discussed earlier. In Appendix II we show that for any $\lambda>0$, the mean reaction time decreases with increasing $v$, that is, MRT decreases as the false-alarm rate increases. provided $I$

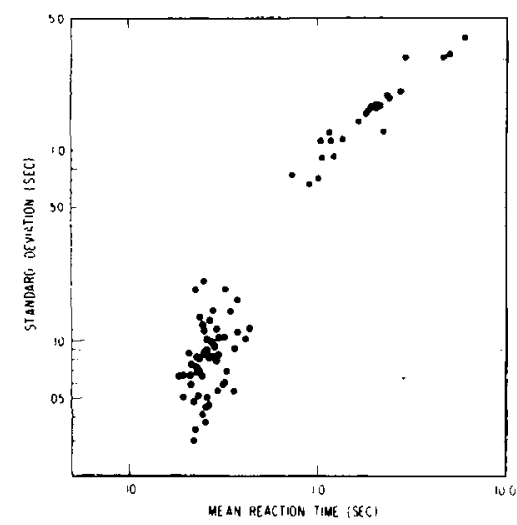

Fig. 15. Standard deviation of reaction-time distribution vs the mean for the various experiments reported in this paper. satisfies a condition stated in the appendix. Most smooth, unimodal, bounded densities meet the condition. Another reasonable possibility is that, except possibly with faint signals, the high rates are produced in a different way from the low ones. One plausible hypothesis is that, during training, Ss first attempted to react to the warning signal, "fast guess," on the requisite number of trials. When we objected to this, as we did, they modified their strategy and time estimated with some fairly broad distribution. Throse estimates that occurred before the signal onset contributed to the false-alasm rate and those after it to the observed reaction-time distribution. If the mean of the latter observations, measured from signal onset, is greater than the actual mean reaction time to the signal, which for intense signals is possible, then we should anticipatc an increase in mean reaction time as this strategy is introduced. In order to increase the false-alarm rate, the $S$ could then reduce the mean of the time estimation distribution. and that will almost certainly entail a reduction in the variance, and so the observed mean reaction time will drop.

Mean Signal Wait and Mean Reaction Time

In this experiment, we held the signal intensity at $54 \mathrm{~dB}$, the false-alarm rate at $5 \%$ and $20 \%$, and varied the mean wait for the signal $(1 / \lambda)$ by successive factors of 2 from $1 / 2$ to $16 \mathrm{sec}$

Our results are generally consistent with those of previous studies, e.g. Nickerson (1967) and Nickerson and Burnham (1969), but there are several differences. Figure 16 shows the data for three Ss. The means are based on about 600 observations for the shortest waits and about 240 for the longest. We have averaged over the two false-alarm rates since there was little difference and what there was did not seem to exhibit any pattern.

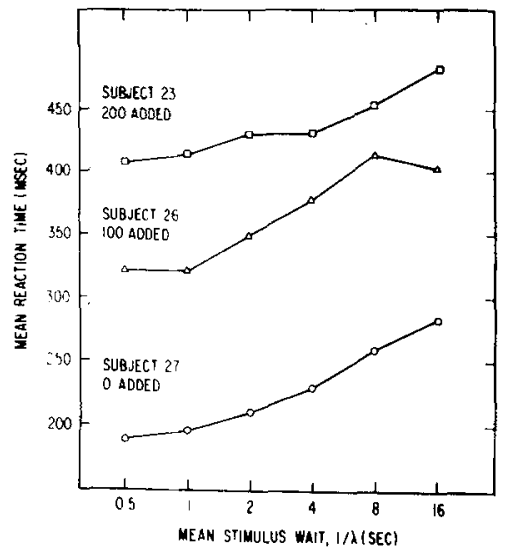

Fig. 16. Mean reaction time as a function of mean stimulus wait. In order to keep the data separate, the curves for two of the three Ss have been displaced on the graph by adding the indicated constant.

In comparing our results with those of previous studies, the rnost obvious difference is that our results show less change in the mean reaction time as a function of the mean wait. Over similar changes in mean wait, Nickerson and Burnham show changes in mean reaction time of about $150 \mathrm{msec}$, from about 270 to $420 \mathrm{msec}$, rather than the change of only 100 msec displayed by our Ss. In their experiment, the reaction signal was a light and the penalty for false alarms was quite heavy. Thus, their false-alarm rates were lower than any we observed and this may account for the somewhat longer response as well as the greater change in mean reaction times as a function of average foreperiods.

Two mechanisms for this effect suggest themselves. First, the actual experimental conditions of different mean waits may underlie it. That is to say, when the $S$ is in an experimental run with a short mean wait, he tends to react more rapidly to all actual waits than when he is in a run with a long mean wait. For example, if on some trial of a run with a mean wait of 1 sec he waits $5 \mathrm{sec}$ for the signal, he will react, on the average, more rapidly to that $5-\mathrm{sec}$ wait than to one embedded in a run with a mean wait of $8 \mathrm{sec}$. Another alternative is that only the actual wait matters, not the condition within which it is embedded. The gist of that hypothesis is that, as the wait for a signal increases, the $\mathrm{S}$ becomes less reactive to its onset; he is at the peak of his preparation at or near the beginning of a trial and, because of fatigue, inattention, or boredom, this state of preparation lessens as he experiences longer and longer waits for the signal onset. According to this hypothesis, there is a single function relating speed of reaction to the duration of the wait until signal onset, 


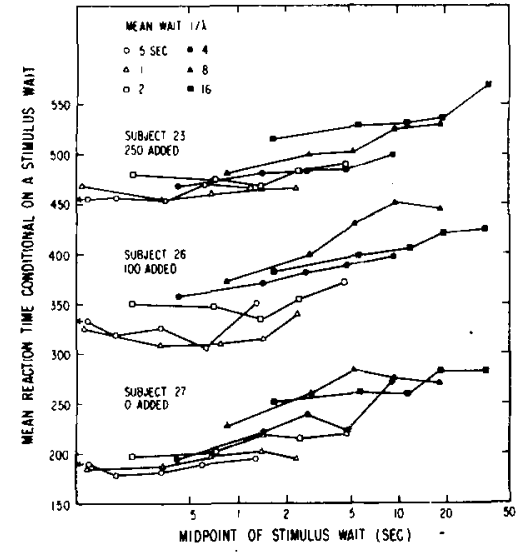

Fig. 17. Mean reaction time given different intervals of stimulus wait from the experiment in which the mean stimulus wait was varied. The mean stimulus wait is given in the key. The data have been displaced on the graph by adding the indicated constant.

and by varying the mean wait we simply sample different ranges of that function. According to the former hypothesis, the distribution of reaction times is the same for any wait within a given condition; we refer to this as the stationarity hypothesis.

According to the latter, the distribution varies with the actual wait; we refer to it as the nonstationarity hypothesis.

As is shown in Appendix II our model predicts the stationarity hypothesis - for waits in excess of $\tau$, which by previous results must be less than $1 / 2 \mathrm{sec}$.

\section{Mean Reaction Time Conditional on Signal Wait}

To examine these alternatives, it is clear that we must group reaction times according to signal waits. To keep sample

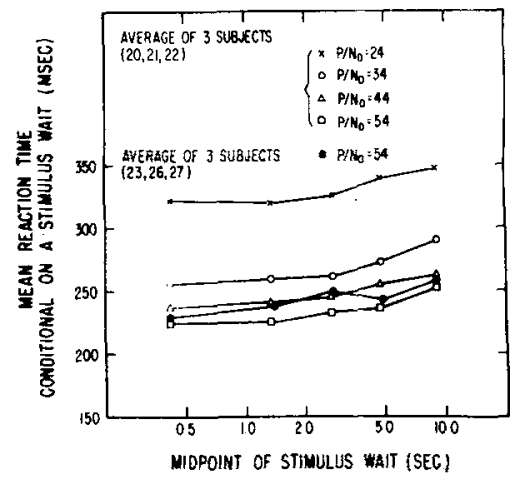

Fig. 18. Mean reaction time given different intervals of stimulus wait for the data reported in Table 2 . The data have been averaged over Ss and false-alarm rates. The solid points are the averages over Ss for the data presented in Fig. 17 for the appropriate mean wait, $1 / \lambda=4 \mathrm{sec}$. sizes reasonable, all we can afford is a division into five intervals which are spaced so that equal numbers of waits are expected in each interval. The data are shown in Fig. 17. There is considerable variability because the points on the left $(1 / \lambda=0.5 \mathrm{sec})$ are based on about 120 observations, whereas those on the right $(1 / \lambda=16 \mathrm{sec})$ are based on as few as 50 . Despite that, it seems clear that both stationary and nonstationary factors are at work. The tendency for each curve to rise suggests a nonstationary factor, whereas the separation of the curves, their height increasing with mean wait, suggests a stationary component. Had stationarity been the only effect, the curves would have been horizontal and nonoverlapping.

Having found the trends shown in Fig. 17, it is interesting to analyze the previous data of this paper in the same way, particularly since this reveals an unanticipated interaction with signal intensity. Figure 18 presents the stimulus-wait analysis for the data presented in Table 2. The data for the 14-dB condition are so far off-scale they are not shown in this figure. These data have been averaged over Ss and false-alarm rates, resulting in about 500 observations per point. The trends, although small, are reliable. Moreover, each $S$ at each false-alarm rate exhibited the same trend. Also included in Fig. 18 are the averaged data for the three Ss shown in Fig. 17 (4-sec mean wait at 54-dB intensity; about 300 observations per point). The trend, while not so stable because of the smaller sample sizes, is still evident. The comparison of the two sets of data, taken under identical conditions (solid circle vs open square), gives some idea of the variability over groups of three Ss.

We omitted from Fig. 18 the data at the

\section{APPENDIX I}

Assuming that $\mu=v+\rho$, Eq. 38 of Luce and Green (1970) is

$$
\text { MRT }=\frac{\bar{r}\left(1+\frac{v}{\lambda}\right)+\frac{1}{v+\rho}-\left(\frac{1-\mathrm{R} \lambda}{\lambda}\right) \frac{v}{\lambda}}{1+v\left(\frac{1-R_{-\lambda}}{\lambda}\right)} .
$$

It follows immediately that

$\frac{\partial M R T}{\partial v}$

$$
=\frac{\frac{1}{\lambda}\left(\bar{r}-\frac{1-R_{-\lambda}}{\lambda}\right)-\bar{r} \cdot\left(\frac{1-R_{-\lambda}}{\lambda}\right)-\left(\frac{1-R_{-\lambda}}{\lambda}\right)\left[\frac{1}{v+\rho}+\frac{v}{(v+\rho)^{2}}\right]-\frac{1}{(v+\rho)^{2}}}{\left[1+v\left(\frac{\left.\left.1-R_{-\lambda}\right)\right]^{2}}{\lambda}\right)\right]^{2}} .
$$

Because $R_{-\lambda}<1$ if

$$
\bar{r}-\frac{1-\mathbf{R}_{-\lambda}}{\lambda}<\overline{\mathbf{r}}\left(1-\mathbf{R}_{-\lambda}\right),
$$

then

$$
\frac{\partial M R T}{\partial v}<0
$$

As is easily seen, this is equivalent to

$$
R_{\cdots \lambda}<\frac{1}{1+\lambda \bar{r}}
$$

Next we show that this inequality is valid for all $\lambda \geqslant 0$ provided that the density, $r$, meets the following condition: For some $t_{0}>0$.

$$
\begin{aligned}
r(t)+\bar{r} r^{\prime}(t) & >0 \text { for } 0 \leqslant t<t_{0} \\
& \leqslant 0 \text { for } t_{0} \leqslant t
\end{aligned}
$$

Note that if $r$ is a smooth. unimodal, and bounded density. this condition is very likely to be met because to the left of the mode both $r$ and $r^{\prime}$ are positive and to the right $r$ decreases and $r$ ' is negative. The only problem is if $r$ approaches 0 , which it will not do if the density is bounded. We assume this condition.

If we let $f(\lambda)=(1+\lambda \bar{r}) R_{\lambda}$, then since $f(0)=1$, it is sufficient to prove 


$$
f^{\prime}(\lambda)=(1+\lambda \bar{r}) R^{\prime} \quad \lambda+\ddot{r} R_{\lambda}<0 \text {. }
$$

Observe that

$$
\begin{aligned}
& \lambda R^{\prime} \quad \lambda=\lambda \int_{1 /}^{\infty} e^{\lambda} \lambda^{\prime} \operatorname{tr}(t) d t \\
& =-\int_{0}^{\infty} e^{\lambda_{1}}|\operatorname{tr}(t)|^{\prime} d t \\
& =-\int_{0}^{\infty} e^{\lambda t}\left[r(t)+t^{\prime}(t) \mid d t\right. \\
& =-\mathbf{R}_{\lambda}-\int_{0}^{\infty} \mathrm{e}^{\lambda t} \operatorname{tr}^{*}(\mathrm{t}) \mathrm{dt} \text {. }
\end{aligned}
$$

Thus,

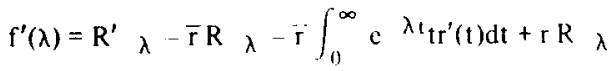

$$
\begin{aligned}
& \left.=\ldots \int_{0}^{1_{0}} e^{\lambda t} t \mid r(t)+\bar{r} r^{\prime}(t)\right] d t \cdot \int_{t_{0}}^{\infty} e^{\lambda} t\left|r(t)+\ddot{r} r^{\prime}(t)\right| d t \\
& \leqslant \mathrm{e}^{\lambda t_{0}} \int_{0}^{\infty} \mathrm{t}\left[\mathrm{r}(\mathrm{t})+\mathrm{r} \mathrm{r}^{\prime}(\mathrm{t})\right] \mathrm{dt} \\
& =\lambda t_{10}\left[1+\int_{0}^{\infty} t r^{\prime}(t) d t\right] \\
& =0 \text {. }
\end{aligned}
$$

\section{APPENDIX II}

We wish to calculate the mean reaction time conditional on a signal onset at time $x$, i.e.,

$$
\operatorname{MRT}(x)=\frac{\int_{0}^{\infty} t P(t, x) d t}{\int_{0}^{\infty} P(t, x) d t}
$$

where

$$
\begin{aligned}
\mathrm{P}(\mathrm{t}, \mathrm{x}) & =\operatorname{Pr}[(\mathrm{R}-\mathrm{S}=\mathrm{t}) \text { and }(\mathrm{t} \geqslant 0) \text { and }(\mathrm{S}=\mathrm{x})] \\
& =\lambda \mathrm{e}^{\lambda} \lambda_{r}\left[\int_{0}^{\lambda} v \mathrm{e}^{v y} \mathrm{r}(\mathrm{t}+\mathrm{x}-\mathrm{y}) \mathrm{dy}+\mathrm{e}^{v \mathrm{x}} \int_{x}^{\lambda+\mathrm{t}} \mu \mathrm{e}^{\mu(\mathrm{y}-\mathrm{x})} \mathrm{r}(\mathrm{t}+\mathrm{x}-\mathrm{y}) \mathrm{dy}\right] \\
& =\lambda \mathrm{e}^{-\lambda} \cdot\left[\mathrm{e}^{v(\mathrm{t}+\mathrm{x})} \int_{t}^{\mathrm{t}+\mathrm{x}} \mathrm{e}^{v z} \mathrm{r}(\mathrm{z}) \mathrm{dz}+e^{v x} \mu \mathrm{e}^{\mu t} \int_{0}^{\mathrm{t}} \mathrm{e}^{\mu z} \mathrm{r}(\mathrm{z}) \mathrm{dz}\right]
\end{aligned}
$$

Substituting this expression into the equation for the mean and integrating by part yields

$$
\operatorname{MRT}(x)=\frac{e^{v \prime} \int_{0}^{\infty} \operatorname{tr}(t+x) d t+\frac{1}{v}\left[\int_{0}^{x} e^{v y} r(y) d y+e^{v x} \int_{x}^{\infty} r(y) d y-1\right]+\frac{1}{\mu}}{e^{v x} \int_{1}^{\infty} r(y) d y+\int_{0}^{x} e^{v y} r(y) d y} .
$$

Recalling that $r(t)=0$ for $t \geqslant \tau$, we see that

$$
\operatorname{MRT}(x)=\frac{\frac{1}{v}\left(R_{v} \cdot 1\right)+\frac{1}{\mu}}{R_{v}}, x \geqslant \tau
$$

and this is independent of $x$.

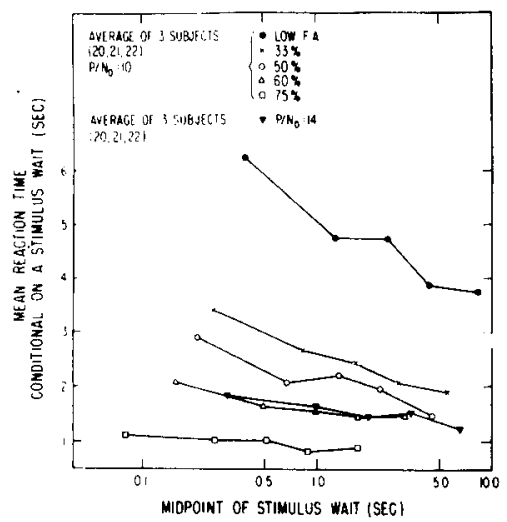

Fig. 19. Mean reaction time given different intervals of stimulus wait for the data taken at low signal-to-noise levels. (The lowest signal level in Table 2 and the data concerning false-alarm rate reported earlier in the paper, Fig. 3.)

lowest signal intensity because it has a different time scale; it also shows a different trend and is greatly affected by false-alarm rate. Figure 19 shows this. Note first the change in scale along the ordinate; we now measure reaction time in seconds, not fractions of a second. In the most extreme case, the low false-alarm rate, the mean reaction time decreases from about $6 \mathrm{sec}$ to about $4 \mathrm{sec}$ as a function of signal wait. In all cases, a decrease is exhibited. These decrements are, however, small in a statistical sense. As seen in Fig. 15, for mean reaction times greater than $1 \mathrm{sec}$, the standard deviation is about the same as the mean, and so all of the changes in Fig. 19 are considerably less than one standard deviation. Since the means are based on at least 200 observations, the change in mean reaction time is statistically significant but clearly not a major variable. Note that the data from another experiment with the same Ss follows the same trend.

In summary, then, all of the data we have collected show that the mean reaction time changes slightly with the length of the signal wait. For intense signals, where detection is not a problem and the reaction times are fast $(\leqslant 300 \mathrm{msec})$, the mean reaction time increases by as much as $30 \%$ from a $1 / 2-\mathrm{sec}$ to a $10-\mathrm{sec}$ signal wait. For difficult-to-detect signals, where the mean reaction time is heavily affected by the detection process, the mean reaction time decreases by about $30 \%-40 \%$ over the same range. In both cases, the effects are not large compared with the variability of reaction limes, and hundreds of observations are required to develop statistical confidence in the trends.

\section{SUMMARY}

Our studies of the reaction-time task can be conveniently divided into strong and 
weak signal cases. For the latter, we find that the false-alarm process can be modeled as an independent additive process which combines with the signal process to cause the initiation of a response. The mean of this process appears to decline somewhat with stimulus wait, but this effect is small compared with the variability. And the ratio of mean to standard deviation is, as one would expect from a Poisson process, about one.

The analytic separation of the observed distribution of reaction times appears interesting and reveals several fundamental weaknesses of the model. First, although the "residual" distribution appears bounded (within $1 / 2 \mathrm{sec}$ ) as postulated in the theory, a large anomaly exists at the bound, namely, the estimated probability "distribution" goes negative, which is absurd. Second, and more fundamental, the distribution determined by analysis of data obtained using weak signals is not the same as the one obtained using high signal-to-noise levels. Thus, the theory does not yet bridge the gap between the detection situation and the conventional, intense-signal, reaction-time task.

For intense signals we find that the mean reaction time increases systematically with signal wait. The conditional signal-wait analysis suggests that the bulk of this increase is due to stationary factors, although some is also caused by nonstationary factors. The size of these effects is small, however, relative to the variability. For intense signals, standard deviation of the reaction time is about $20 \%$ of the mean. This change in the ratio of the mean to the standard deviation from about 1 to 5 suggests quite different processes are at work, a view which is fundamental to the analytic model.

\section{REFERENCES}

GREEN, D. M., \& LUCE, R. D. Detection of auditory signals presented at random times. Perception \& Psychophysics, 1967, 2, 441-450.

GRICE, R. C. Stimulus intensity and response evocation. Psychological Review, 1968, 75, 359-373.

KAISER, J. F. Digital filters. In F. K. Kuo and J. F. Kaiser (Eds.), System analysis by digital computer. New York: Wiley, 1966.

KOHFELD, D. L. Stimulus intensity and adaptation level as determinates of simple reaction time. Journal of Experimental Psychology, 1968, 76, 468-473.

LAMMING, D. R. J. Information theory of choice-reaction times. London and New York: Academic Press, 1968.

LUCE, R. D., \& GREEN, D. M. Detection of auditory signals presented at random times, II. Perception \& Psychophysics, 1970, 7, 1-14.

McGILL, W. J. Loudness and reaction time. Acta Psychologica, 1961, 19, 193-199.

McGILL, W. J., \& GIBBON, J. The general gamma distribution and reaction times. Journal of Mathematical Psychology, 1965, 2, 1-18.

NICKERSON, R. S. Expectancy waiting time and the psychological refractory period. Acta Psychologica, 1967, 27, 23-34.
NICKERSON, R. S., \& BURNHAM, D. W Response times with nonaging foreperiods Journal of Experimental Psychology, 1969 79, $452-457$.

OLLMAN, R. Fast guesses in choice reactior times. Psychonomic Science, 1966, 6 155-156.

STERNBERG, S. The discovery of processin stages: Extensions of Donders' method. In W G. Koster (Ed.), Attention \& performance II Acta Psychologica, 1969, 30, 276-315.

STONE, M. Models for choice-reaction time Psychometrika, 1960, 25, 251-260.

YELLOTT, J. I., JR. Correction for guessing ir choice reaction time. Psychonomic Science $1967,8,321-322$

\section{NOTES}

1. In our earlier papers and in several talks, ws spoke of this as the "reaction latency." This wa: inadvertently misleading because it appeared to imply that this latency occurred only after the decision process had concluded and involved largely, motor delay. In fact, a theory such a: ours predicts the same distribution of reactio times if the various stages are reordered. A mola theory of stages often has no way to specify the order in which the various latencies occu. (Sternberg, 1969). We hope the new terminologs will emphasize that $r(t)$ refers to all those aspect of the reaction-time distribution not treated ir the discussion of the sensory-decision process.

2. A Hamming window is a symmetric positiv function having nonzero values only over a finit interval.

$$
\mathrm{D}(\tau)=0.54+0.46 \cos \pi \tau / \mathrm{T}_{\mathrm{m}}|\tau|<\mathrm{T}_{\mathrm{m}}
$$

where $\tau=k \Delta t$ and $k=0, \pm 1, \pm 2, \cdots \pm_{n}$ anc $\Delta \mathrm{t}=\mathrm{T}_{\mathrm{m}} / \mathrm{n}$.

(Accepted for publication July 29, 1970.) 